\title{
Investigation of the Flow in a Diffusive S-Duct Inlet with and without Secondary Flow Control
}

\author{
L. F. Zhang ${ }^{1 *}$, Z. X. Liu ${ }^{1}$, D. M. Guo ${ }^{1}$, X. F. Wang ${ }^{2}$ \\ ${ }^{1}$ School of Power and Energy, Northwestern Polytechnical University, Xi'an 710072,China \\ ${ }^{2}$ Chinese Flight Test Establishment, Xi'an 710089, China \\ Email: zlfnwpu@mail.nwpu.edu.cn
}

\begin{abstract}
A computational study of the flow characteristics inside a diffusive S-duct inlet with and without vortex generators (VGs) is conducted. The primary discussion herein is focused on the development of secondary flow in the S-duct, pressure recovery and distortion at the exit for the S-duct with and without VGs. The S-duct has a cross-sectional area change from a retangular at the entrance to a full circle at the exit. Full three-dimensional Navier-Stokes equations are solved using finite volume method and $\kappa-\varepsilon$ turbulence model is employed. In order to validate the numerical methods, the predicted results of surface pressure are compared with flight test for S-duct without VGs. And it shows fairly good agreement. The CFD computed flows in the S-duct and out of the S-duct inlet are carefully examined.

Cross-sectional area change or curvature of the duct centerline lead to streamline curvature. The cross stream pressure gradients resulting from steamline curvature induce significant secondary flow. It is a dominant feature for S-duct. Six cross-sectional planes reveal the development of secondary flow in the S-duct with and without VGs. The different secondary flow developing progresses between S-duct with and without VGs are discussed.

A large pair of counter-rotating vortices caused by secondary flow is at the exit for the S-duct without VGs. Low momentum fluid of the boundary layer are convected to the center of the duct. High momentum and low momentum fluid is blended downstream, so that both the uniformity and magnitude of the total pressure profile are degraded.

Performance of the S-duct with two different location VGs is assessed by calculating total pressure recovery and distortion, and comparing them to the values for the S-duct without VGs. Through the comparison, it shows that the installation of VGs in the S-duct inlet at two different locations is helpful for decreasing distortion and swirl, but it is ineffective in improving the pressure recovery. Counter-rotating vortices are weakened to different extent at the exit in the S-duct with two different location VGs.
\end{abstract}

\section{REFERENCES}

1. Jirásek A. Development and application of design strategy for design of vortex generator flow control in inlets. AIAA 2006-1050

2. Mohler S R. WIND-US flow calculations for the M2129 S-duct using structured and unstructured grids. AIAA-2004-0525

3. Harloff G J, Reichert B A, Wellborn S R. Navier-stokes analysis and experimental data comparison of compressible flow in a diffusing S-duct. AIAA-92-2699 